\title{
Activité Antioxydante des Extraits Méthanoliques de Differents Organes de Detarium microcarpum Guill. \& Perr.
}

\author{
Hama Hamadou Habibou, PhD \\ Moussa Idrissa, MC \\ Ikhiri Khalid, Pr
}

Université Abdou Moumouni, Faculté des Sciences et Techniques, Département de Chimie, Laboratoire des Substances Naturelles et Synthèse Organique (LASNASO), Groupe de Recherche Substances Naturelles et Valorisations, Niamey, Niger

\section{Ouedraogo Benjamin, PhD}

Université Ouaga I Joseph KI-ZERBO, Laboratoire de Chimie Analytique, Environnementale et Bio-organique (LCAEBiO), Département de Chimie,

Unité de Formation et de Recherche en Science Exactes et Appliquées, Ouagadougou-Burkina Fasso

\section{Adamou Rabani, Pr}

Université Abdou Moumouni, Faculté des Sciences et Techniques, Département de Chimie, Laboratoire Matériaux, Eaux et Environnement (LAMEE), Niamey, Niger

Doi: 10.19044/esj.2019.v15n12p159 URL:http://dx.doi.org/10.19044/esj.2019.v15n12p159

\section{Résumé}

Une grande partie de l'intérêt des recherches actuelles porte sur l'étude de molécules antioxydantes d'origine naturelle. Cette étude vise à évaluer l'activité antioxydante des extraits méthanoliques de différents organes de Detarium microcarpum : écorces de tronc et de racine, feuilles, fruits et fleurs. La teneur en polyphénols totaux a été déterminée par la méthode de FolinCiocalteu et l'activité antioxydante par deux méthodes : le piégeage des radicaux libres du DPPH et la méthode de réduction du fer FRAP (Ferric reducing antioxidant power). Les teneurs en polyphénols totaux des différents organes varient de 109,44 à 40,06 mg équivalents acides galliques par gramme d'extrait (mg EAG/g). La capacité de réduction de fer est remarquable dans l'extrait des écorces du tronc $(171,71 \mathrm{~mol} \mathrm{EAA} / 100 \mathrm{mg})$; de racine $(170,79$ mol EAA/100mg) et des feuilles (127,96 mol EAA/100mg). La capacité de piégeage du DPPH a montré une réduction du radical DPPH avec des concentrations inhibitrices $\mathrm{CI}_{50}$ de 0,225 à $0,75 \mathrm{mg} / \mathrm{ml}$ mais restent toujours 
faibles par rapport au Trolox (antioxydant de référence, $0,05 \mathrm{mg} / \mathrm{ml}$ ). L'activité antioxydante des extraits de Detarium microcarpum mise en évidence dans cette étude pourrait justifier les usages traditionnels de cette plante afin de développer de nouveaux composés bioactifs.

Mots-clés : Detarium microcarpum, DPPH, FRAP, activité antioxydante

\title{
Antioxidant Activity of Méthanolic Extracts from Various Organs of Detarium microcarpum Guill.\& Perr.
}

\author{
Hama Hamadou Habibou, PhD \\ Moussa Idrissa, MC \\ Ikhiri Khalid, Pr
}

Université Abdou Moumouni, Faculté des Sciences et Techniques, Département de Chimie, Laboratoire des Substances Naturelles et Synthèse Organique (LASNASO), Groupe de Recherche Substances Naturelles et

Valorisations, Niamey, Niger

\section{Ouedraogo Benjamin, PhD}

Université Ouaga I Joseph KI-ZERBO, Laboratoire de Chimie Analytique, Environnementale et Bio-organique (LCAEBiO), Département de Chimie,

Unité de Formation et de Recherche en Science Exactes et Appliquées,

Ouagadougou-Burkina Fasso

Adamou Rabani, Pr

Université Abdou Moumouni, Faculté des Sciences et Techniques, Département de Chimie, Laboratoire Matériaux, Eaux et Environnement (LAMEE), Niamey, Niger

\begin{abstract}
Many of the current research interest include the study of molecular antioxidant compounds of natural source. This study aims to evaluate the antioxidant activities of the methanolic extracts of different parts of Detarium microcarpum: trunk and root bark, leaves, fruits and flowers. The total polyphenol content was determined by the Folin-Ciocalteu method and the antioxidant activity by two methods: the free radical scavenging of the DPPH and the Ferric reducing antioxidant power (FRAP) method. The total polyphenol content of plant parts was found to vary from 109.44 to $40.06 \mathrm{mg}$
\end{abstract}


gallic acid equivalents per gram of extract (mg EAG/g). Iron reduction capacity was found to be highest in the trunk bark (171.71 mole EAA/100mg); root (170.79 mole EAA/100mg) and leaves (127.96 mole EAA/100mg) extracts. The DPPH scavenging capacity showed a significant reduction of the $\mathrm{DPPH}$ radical with $\mathrm{IC}_{50}$ inhibitory concentrations ranging from 0.225 to 0.75 $\mathrm{mg} / \mathrm{ml}$ but still remain low compared to that of Trolox as standard antioxydant. The antioxidant activities of Detarium microcarpum extracts reported in this study could justify the traditional uses of this plant in order to develop new bioactive compounds.

Keywords: Detarium microcarpum, DPPH, FRAP, antioxidant activity

\section{Introduction}

L'usage de la médecine traditionnelle est très répandu en Afrique. Les plantes médicinales ont toujours eu une place importante dans l'arsenal thérapeutique de l'humanité. Selon l'Organisation Mondiale de la Santé (OMS), environ 65 à $80 \%$ de la population dans les pays en développement, dépendent essentiellement des plantes médicinales traditionnelles pour leurs soins de santé primaire (OMS, 2002).

Le stress oxydatif est impliqué dans un large spectre de maladies qui ont un impact énorme sur la santé des populations. Le métabolisme aérobie chez les mammifères génère des substances appelées espèces réactives de l'oxygène (ERO) qui sont impliquées dans des processus physiologiques (Favier, 2003).

Cependant l'excès de production des ERO peut devenir toxique pour les composants majeurs de la cellule, les lipides, les protéines et l'acide nucléique et par conséquent donné lieu au stress oxydatif (Valko et al., 2006). Ce dernier est impliqué dans diverses pathologies telles que les maladies cardiovasculaires, les cancers, le diabète etc (Aruoma, 2003). Un antioxydant est défini comme toute substance capable, à concentration relativement faible, d'entrer en compétition avec d'autres substrats oxydables et ainsi retarder ou empêcher l'oxydation de ces substrats (Berger, 2006). De nombreuses études ont montré que les plantes possèdent des propriétés antioxydantes dues en grande partie à leurs composés phénoliques.

Ils peuvent prévenir la modification oxydative par neutralisation des radicaux libres, piégeage de l'oxygène ou décomposition des peroxydes par l'intermédiaire de leurs activités antioxydantes (Yemeda et al., 2014).

Parmi des nombreuses plantes de la riche flore africaine, figure le Detarium microcarpum Guill. \& Perr. Il est employé en médecine traditionnelle pour le traitement de nombreuses maladies et fait partie de plusieurs pharmacopées locales en Afrique de l'Ouest. 
Le décocté des écorces de tronc est employé, en boisson pour guérir la diarrhée simple ou sanguinolente. Il serait antihémorroïdique et antiblennorragique (Adama, 1997) ; antimicrobien (Kouyaté et Van Damme, 2002 ; Loubaki et al., 1991) ; anti-œdémateux (Ikhiri et Ilagouma, 1995). Le macéré des racines est utilisé contre les maux de ventre et les diarrhées dysentériques. Le décocté des racines exhalerait un parfum agréable; le liquide ainsi obtenu, s'emploie en boisson contre la syphilis (Koyaté., 2005). Les racines présentent des propriétés antidiabétiques (Okolo et al., 2012) et antifongiques (Adamu et al., 2014).

Le décocté des feuilles de Detarium microcarpum est utilisé pour soigner les maux de ventre, la carie dentaire, les complications d'accouchement (Kouyaté, 2015). Elles présentent des propriétés antidiarrhéiques et anti-asthéniques (Okolo et al., 2012), antimicrobiennes (Ebi et al., 2011) et larvicides (Adebote et al.,2006).

Les fruits jouent un rôle important au niveau nutritionnel selon plusieurs travaux effectués sur leur composition nutritionnelle et thérapeutique (Cavin et al., 2006 ; Felix et al., 2010 ; Rouamba et al., 2017).

Des travaux antérieurs réalisés sur différents organes de $D$. microcarpum ont montré qu'ils contenaient en majorité des composés phénoliques ayant un fort potentiel antioxydant corroborant leurs usages traditionnels (Hama et al., 2018). Les polyphénols suscitent depuis une dizaine d'année un intérêt croissant de la part des nutritionistes, des industriels de l'agro-alimentaire et des consommateurs. Une des raisons principales est la reconnaissance de leurs propriétés antioxydantes et ainsi leur implication probable dans la prévention des diverses pathologies associées au stress oxydant (Mamta et al., 2013). A notre connaissance, aucune étude n'a été réalisée sur le potentiel antioxydant de Detarium microcarpum, d'où l'objectif de cette étude qui est d'évaluer l'activité antioxydante des extraits méthanoliques de Detarium microcarpum.

\section{Matériel et méthodes}

\section{Matériel végétal et extraction}

Les organes (feuilles, fruits, fleurs et écorces du tronc et de racine) $\mathrm{du}$ Detarium microcarpum ont été fraichement récoltés au mois de mars 2017 dans la région de Dosso (Niger). Ils ont été identifiés au Département de Biologie (Faculté des Sciences et Techniques de l'Université Abdou Moumouni). Le matériel végétal a été séché à l'air libre à l'abri du soleil et à la température ambiante, puis réduit en poudre.

$30 \mathrm{~g}$ de poudre végétale de chaque organe de Detarium microcarpum ont été macérés dans $300 \mathrm{~mL}$ (×2) de méthanol. Après une extraction continue à la macération pendant $24 \mathrm{~h}$ à la température ambiante sous agitation 
magnétique, le macérât est filtré et le méthanol a été éliminé par évaporation. au rotavapor.

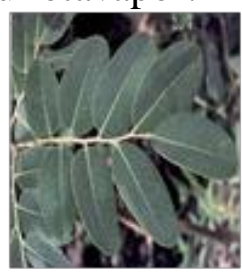

Les feuilles

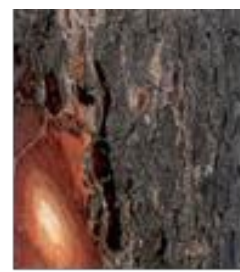

L'écorce du tronc

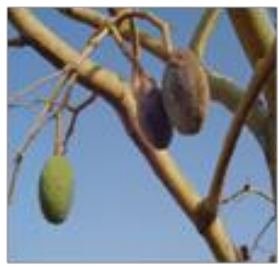

Les fruits

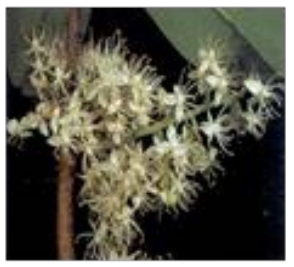

Les fleurs

Figure 1: Les différents organes du Detarium microcarpum

\section{Dosage spectrophotométrique des polyphénols totaux}

Les teneurs totales en composés phénoliques des extraits méthanoliques de différents organes de Detarium microcarpum ont été estimées en utilisant le réactif de Folin Ciocalteau (Siddiqui et al., 2017). A l'extrait méthanolique de concentration $1 \mathrm{~g} / \mathrm{L}$ de chaque organe de Detarium microcarpum, on additionne $5 \mathrm{~mL}$ de réactif Folin Ciocalteu dilué 10 fois et 4 $\mathrm{mL}$ de solution de carbonate de Sodium (75 g/L). Le mélange est agité et incubé à l'obscurité à température ambiante pendant 30 minutes et l'absorbance a été mesurée à $760 \mathrm{~nm}$ en utilisant un spectrophotomètre UV (thermo scientific, evolution 300). La courbe d'étalonnage a été tracée en utilisant $1 \mathrm{~mL}$ de solution d'acide gallique de concentrations : 0, 20, 40, 60 et $80 \mathrm{mg} / \mathrm{L}$. Les résultats sont exprimés en $\mathrm{mg}$ équivalent acide gallique/g d'extrait (mg EAG/g) en se référant à la courbe d'étalonnage de l'acide gallique. L'absorbance a été mesurée pour déterminer les teneurs en polyphénols totaux en utilisant l'Équation (1) :

$$
\mathrm{C}=\frac{\mathrm{C}_{1} \times \mathrm{V}}{\mathrm{m}}
$$

$\mathrm{C}$ étant la teneur en polyphénols totaux exprimée en mg équivalent acide gallique/g d'extrait, $\mathrm{C}_{1}$ la concentration d'acide gallique établie à partir de la courbe d'étalonnage en $\mathrm{mg} / \mathrm{L}, \mathrm{V}$ le volume d'extrait en $\mathrm{L}$ et $\mathrm{m}$ le poids de l'extrait de plante en $\mathrm{g}$.

\section{Activité antioxydante in vitro}

Test du pouvoir de réduction du fer : FRAP (Ferric reducing antioxidant power) :

Principe

Le pouvoir réducteur d'un extrait est associé à son pouvoir antioxydant. Cette technique a été développée pour mesurer la capacité des extraits à réduire le fer ferrique $\left(\mathrm{Fe}^{3+}\right)$ présent dans le complexe $\mathrm{K}_{3} \mathrm{Fe}(\mathrm{CN})_{6}$ en fer ferreux $\left(\mathrm{Fe}^{2+}\right)$. Le fer ferrique initialement jaune, se réduit et devient bleu 
ou vert en présence d'un atome d'électron. Le changement de la coloration de jaune à bleu ou vert est proportionnel à l'activité antioxydante.

$$
\mathrm{Fe}^{3+}+\mathrm{e}^{-} \longrightarrow \mathrm{Fe}^{2+}
$$

\section{Protocole}

Le pouvoir réducteur des échantillons a été évalué selon la méthode spectrophotométrique décrite par Rakiatou (2014). Dans un tube à essai contenant $0,5 \mathrm{~mL}$ de solution d'échantillon $(1 \mathrm{mg} / \mathrm{mL})$ est ajouté $1,25 \mathrm{~mL}$ de tampon phosphate $(0,2 \mathrm{M}, \mathrm{pH} 6,6)$ puis $1,25 \mathrm{~mL}$ de potassium hexacyanoferrate $\left[\mathrm{K}_{3} \mathrm{Fe}(\mathrm{CN})_{6}\right] 1 \%$ dans $1^{\prime}$ 'eau. L'ensemble est chauffé à $50^{\circ} \mathrm{C}$ au bain-marie pendant 30 minutes. 1,25 mL d'acide trichloroacétique (10\%) est ensuite ajouté. Trois aliquotes de $0,625 \mathrm{~mL}$ sont effectués dans 3 tubes auxquels est ajouté $0,625 \mathrm{~mL}$ d'eau distillée puis $0,125 \mathrm{~mL}$ de $\mathrm{FeCl}_{3} 1 \%$ fraîchement préparé dans l'eau. Un blanc sans échantillon est préparé dans les mêmes conditions.

La lecture est faite à $700 \mathrm{~nm}$ contre une courbe-étalon d'acide ascorbique. La concentration en composés réducteurs (antioxydants) dans l'extrait est exprimée en mol Equivalent Acide Ascorbique par cent gramme d'extraits secs (mol EAA/100g) selon la formule suivante :

$$
\mathrm{C}=\frac{c \times D}{M \times c i} \times 100
$$

$\mathrm{C}=$ concentration en composés réducteurs en mol EAA/100g d'extrait sec

$\mathrm{c}=$ concentration de l'échantillon lue $(\mathrm{mg} / \mathrm{mL})$

$\mathrm{D}=$ facteur de dilution de la solution mère d'extrait

$\mathrm{Ci}=$ concentration de la solution mère d'extrait $(\mathrm{mg} / \mathrm{mL})$

$\mathrm{M}=$ masse molaire de l'acide ascorbique (176 g/mol)

\section{Test de réduction du radical DPPH}

\section{Principe}

La méthode de mesure du pouvoir antioxydant par le DPPH (2,2Diphényl-picrylhydrazine) repose sur la capacité d'un composé à réduire le radical $\mathrm{DPPH}^{\circ}$. La réduction se traduit par un changement de couleur de la solution qui vire du violet au jaune en présence d'un composé antiradicalaire. La réaction est alors quantifiée en mesurant l'absorbance de la solution par spectrophotométrie à $490 \mathrm{~nm}$. Le changement de la coloration du violet au jaune est proportionnel au pouvoir antioxydant. 


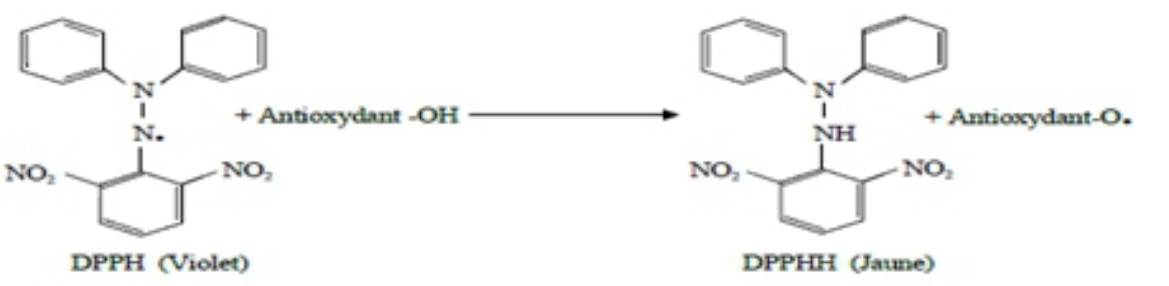

Figure 2: Réduction du DPPH par un antioxydant

\section{Protocole}

La capacité des extraits à réduire les radicaux libres de la DPPH a été déterminée par la méthode spectrophotométrique décrite par Rakiatou (2014). Ainsi, 4mg de DPPH ont été dissout dans $100 \mathrm{~mL}$ de méthanol et $50 \mathrm{mg}$ de chaque échantillon dans $10 \mathrm{~mL}$ de DMSO $(5 \mathrm{mg} / \mathrm{mL})$. Une série de dix (10) dilutions successives a été réalisée sur une microplaque de 96 puits, à partir de la solution mère $(5 \mathrm{mg} / \mathrm{mL})$ des échantillons. Une plaque test a été préparée selon la même configuration que la plaque de la gamme de dilution de la substance à étudier. Le trolox a servi de témoin pour l'évaluation de l'activité antioxydante. Après 30 minutes d'incubation, l'absorbance est lue à $490 \mathrm{~nm}$ contre un blanc à l'aide d'un spectrophotomètre UV-VIS et la concentration nécessaire pour dégrader $50 \%$ du radical DPPH $\left(\mathrm{CI}_{50}\right)$ a été déterminée.

\section{Traitement des données}

L'analyse des résultats a été réalisée par Microsoft@ Office Excel 2010 et Microcal Origin 6.0 Professional pour les graphes.

\section{Résultats et discussion}

\section{Teneurs en polyphénols totaux}

Les teneurs en polyphénols totaux en $\mathrm{mg}$ EAG/g d'extrait sec sont présentées en Figure 3. Ces teneurs varient de 109,44 à 40,06 mg EAG/g d'extrait sec. Les fortes teneurs en polyphénols sont observées dans les écorces du tronc (109,44 mg EAG/g) suivis des écorces de racine (106,88 mg EAG/g) et des feuilles (105,00 mg EAG/g). 


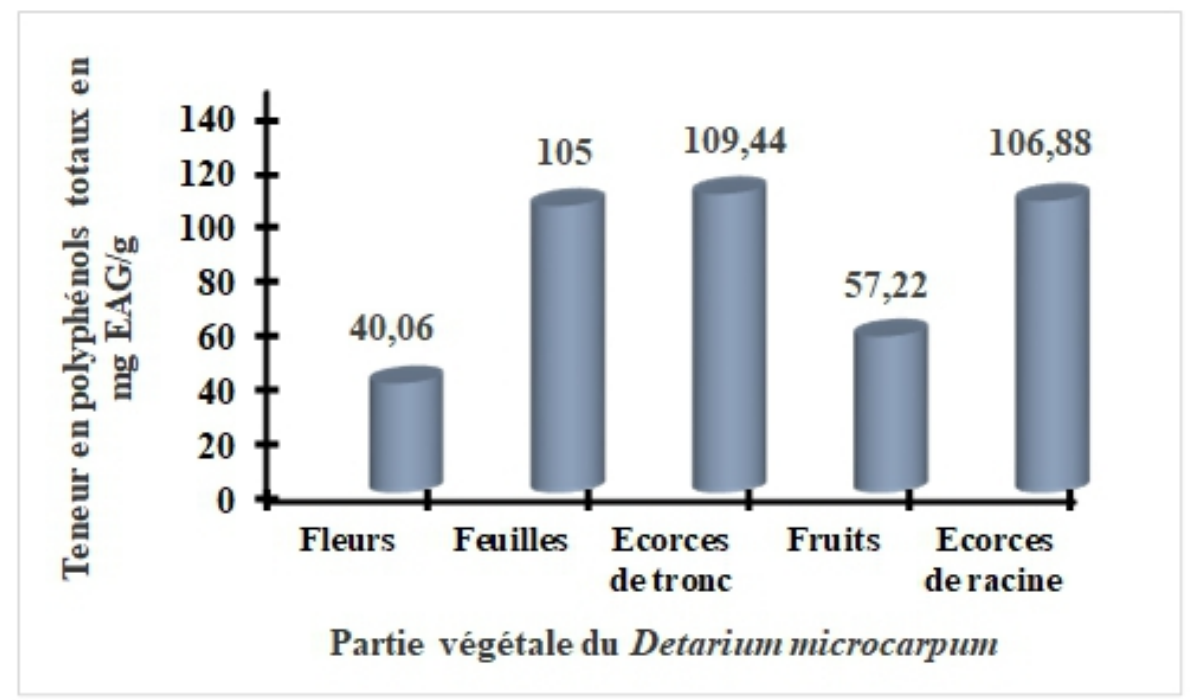

Figure 3 : Teneurs en polyphénols totaux des organes de D. microcarpum

Les résultats montrent que les composés polyphénoliques sont abondants dans les écorces du tronc et de racine et les feuilles. La teneur élevée en polyphénols est liée à la solubilité élevée des phénols dans les solvants polaires (Ghedadba et al., 2014). Le métabolite secondaire peut changer pendant le développement de la plante ; aussi aux conditions climatiques dures (la température élevée, exposition solaire, sécheresse, salinité), qui stimulent la biosynthèse des métabolites secondaires tels que les polyphénols (Falleh et $a l ., 2008)$. En plus de l'organe analysé, la région et la date de la récolte et les solvants qui sont utilisés (Tirichine, 2010).

En effet, la teneur phénolique d'une plante dépend d'un certain nombre de facteurs intrinsèques (génétique) et extrinsèques (les pratiques culturelles, la maturité à la récolte et les conditions de stockage) (Mounira, 2015). En plus, la méthode de quantification peut également influencer l'estimation de la teneur des phénols totaux (Lee et al., 2003).

La variabilité des teneurs en polyphénols est probablement due à la composition phénolique des extraits, aux conditions biotiques telles que l'espèce, la plante hôte, l'organe ou l'état physiologique et aux conditions abiotiques (saison, climat et température) (Mounira, 2015). 


\section{Activité antioxydante par FRAP}

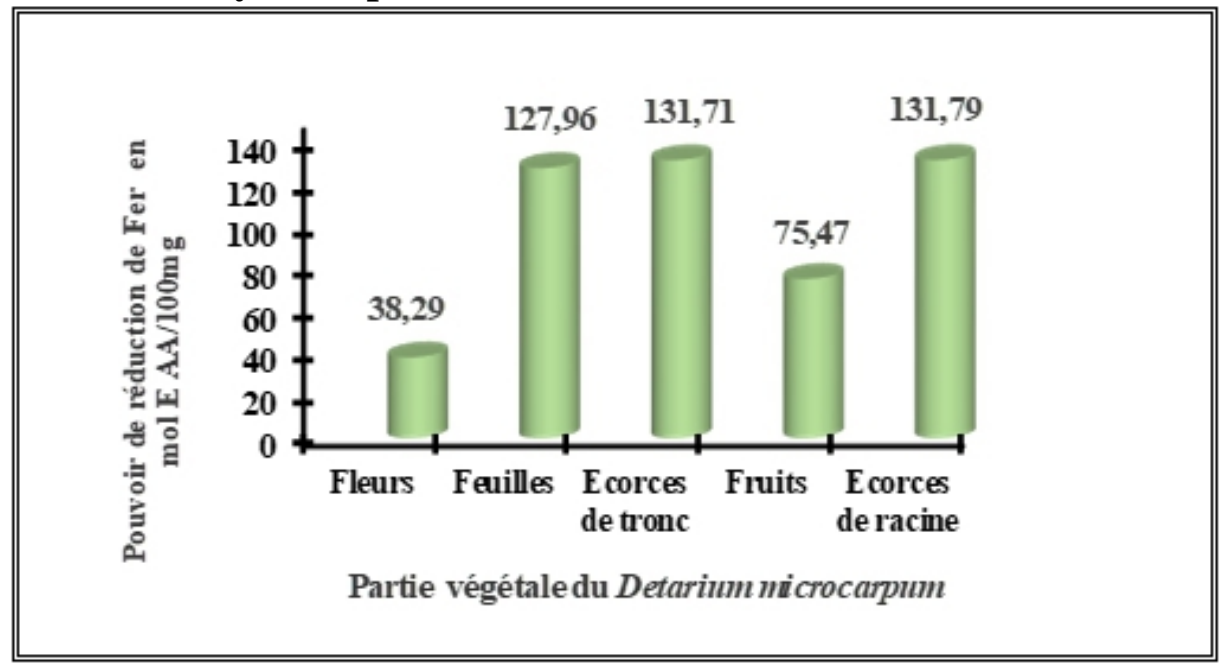

Figure 4 : Pouvoir de réduction de Fer des organes de D. microcarpum

Les propriétés réductrices des extraits de $D$. microcarpum ont été évaluées en utilisant la méthode de FRAP. Elle est basée sur la capacité des extraits à réduire le fer ferrique $\mathrm{Fe}^{3+}$ en fer ferreux $\mathrm{Fe}^{2+}$. Les résultats obtenus montrent que tous les extraits des différents organes ont un pouvoir de réduire le fer et la capacité de réduction est presque identique pour les feuilles, les écorces du tronc et de racine (Figure 4). La forte activité de l'extrait des feuilles, des écorces de tronc et de racine serait due à sa forte teneur en composés polyphénoliques. Ces résultats corroborent les travaux de Yemeda et al. (2014) qui trouvent que les extraits méthanoliques de tiges et des rameaux de Phragmanthera capitata sont plus réducteurs que les extraits de fruits et de fleurs. Ces activités sont corrélées aux teneurs en polyphénols totaux dans les organes impliqués.

\section{Activité antioxydante par DPPH}

Tableau I : Valeurs des $\mathrm{CI}_{50}$

\begin{tabular}{l|llllll}
\hline \hline Extraits & $\begin{array}{l}\text { Ecorces } \\
\text { de tronc }\end{array}$ & $\begin{array}{l}\text { Ecorces de } \\
\text { racine }\end{array}$ & Feuilles & Fruits & Fleurs & Trolox \\
\hline \hline$I C_{50}(\mathrm{mg} / \mathrm{ml})$ & $\mathbf{0 , 0 7 5}$ & $\mathbf{0 , 0 9 7}$ & $\mathbf{0 , 1 4 2}$ & $\mathbf{0 , 2 4 6}$ & $\mathbf{0 , 2 7 1}$ & $\mathbf{0 , 0 5}$
\end{tabular}

La concentration inhibitrice $\mathrm{CI}_{50}$ est inversement proportionnelle à la capacité antioxydante d'un composé, elle exprime la quantité d'antioxydant requise pour diminuer la concentration du radical libre de $50 \%$. Plus la valeur de la $\mathrm{CI}_{50}$ est petite, plus l'activité antioxydante d'un composé est grande (Khoudali et al., 2014). Les valeurs de $\mathrm{CI}_{50}$ de l'extrait méthanolique de Detarium microcarpum sont représentées dans le tableau I. L'extrait 
méthanolique de Detarium. microcarpum rend le radical libre stable (2.2 diphenyl-1-picrylhydrazyl) au diphenyl-picrylhydrazine jaune-coloré avec une $\mathrm{CI}_{50}$ de $0,075 \mathrm{mg} / \mathrm{mL}$ pour les écorces du tronc et $0,097 \mathrm{mg} / \mathrm{mL}$ pour les écorces de racine montrant une activité antioxydante très importante mais restant faibles à celle du trolox qui ramène la stabilité au DPPH avec une $\mathrm{CI}_{50}$ de $0,050 \mathrm{mg} / \mathrm{mL}$. D'après ces résultats, le trolox reste $l^{\prime}$ antioxydant le plus efficace par rapport à l'extrait méthanolique des organes de la plante étudiée.

\section{Correlation des teneurs en polyphénols totaux et l'activité antoxydante}

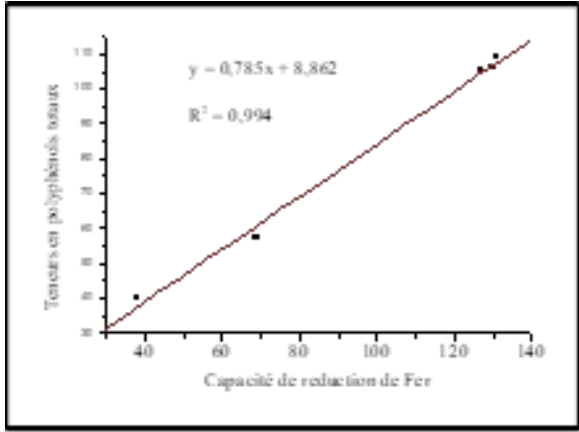

(a)

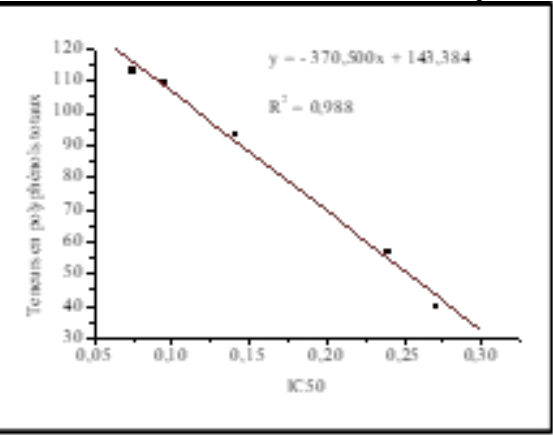

(b)

Figure 5 : Corrélation entre les teneurs en polyphénols totaux et l'activité antioxydante

Les activités antioxydantes sont corrélées aux teneurs en polyphénols totaux dans les organes du Detarium microcarpum. L'activité antioxydante attribuée aux polyphénols s'explique en partie par leur capacité à capturer des radicaux libres et de complexer des métaux (Bahorun et al., 1996).

\section{Conclusion}

La présente étude a permis de mettre en évidence les teneurs en polyphénols totaux et l'activité antioxydante des extraits méthanoliques de différents organes de Detarium microcarpum. Selon les résultats obtenus dans cette étude, la plante est une source potentielle de polyphénols possédants des propriétés antioxydantes d'origine naturelle qui justifient son utilisation traditionnelle dans le traitement de nombreuses affections lié au stress oxydatif. Les extraits méthanoliques des écorces du tronc et de racine ont presenté les meilleurs teneurs en polyphénols et une très grande activité de piégeage des radicaux libres du DPPH et une forte capacité de reduction du fer. Ces résultats pourraient constituer une base scientifique solide pour la recherche de nouveaux composés ayant une importante application dans les industries pharmaceutiques et alimentaires. 


\section{Remerciements}

Nous remercions M. Moussa SOULEY, technicien de laboratoire au département de Chimie de la Faculté des Sciences et Technique, Université Abdou Moumouni et M. Arzika TANIMOUNE technicien de laboratoire de Biologie de la Faculté des Sciences et Technique, Université Abdou Moumouni.

\section{References:}

1. Adama, O. (1997). L'effet de la coupe de Detarium microcarpum Guill, \& Perr. sur la régénération de la végétation dans la forêt classée de Nazinon. Mémoire d'ingenieur, Université de Ouagadougou, (Burkina Fasso), 108 p.

2. Adamu, H.M., Ushie, Longbap B.D., Kuburat, R.J. \& Lawal, U. (2014). Bioactivity of active extracts of the root and stem bark of (Detarium microcarpum). Scientia Research Library. Journal of Applied Chemistry, 2 (2), 31-39.

3. Adebote, D.A., Abolude, D.S., Oniye, S.J., Olododo S.S., \& Hassan, M.M. (2006). Larvicidal and repellent actions of Detarium microcarpum seeds oil against the larvae of Dermestes lardarius (coleopteran: Dermestidae) in dried Clarias gariepinus fish. Journal of entomology, 3(3), 248-253.

4. Aruoma, O.I. (2003). Methodological considerations for characterizing potential antioxidant actions of bioactive components in food plants. Journal of Mutation Research, 9(20), 523 - 524.

5. Bahorun, T., Gressier B., Trotin, F., Brunet, C., Dine, T., Luyckx, M., Vasseur, J., Cazin, M., Cazin, J.C., Pinkas, M. (1996). Oxygen species scavenging activity of phenolic extracts from hawthorn fresh plant organs and pharmaceutical preparations. Arznei. Forschung, 46, 10861089.

6. Berger, M.M. (2006). Manipulations nutritionnelles du stress oxydant : état des connaissances. Nutrition Clinique et Métabolisme, 20, 48-53.

7. Cavin, A. L., Hay, A. E., Marston, A., Stoeckli-Evans, H., Scopelliti, R., Diallo, D. \& Hostettmann, K. (2006). Bioactive diterpenes from the Fruits of Detarium microcarpum. Journal of natural products, 69(5), 768-773.

8. Ebi, G.C and Afieroho, O.E. (2011). Phytochemical and antimicrobial studies on Detarium microcarpum Guill and Sperr (Caesalpinioceae) seeds coat. African Journal of Biotechnology, 10(3), 457-462.

9. Falleh, H., Ksouri, R., Chaieb, K., Karray-Bouraoui N., Trabelsi N., Boulaaba, M., Abdelly, C., (2008). Phenolic composition of Cynara cardunculus L. organs, and their biological activities, Comptes Rendus Biologies, 331, 372-379. 
10. Favier, (2003). Le stress oxydant. Intérêt conceptuel et expérimental dans la compréhension des mécanismes des maladies et potentiel thérapeutique. L'actualité chimique, 108-115.

11. Félix K.S., Ouédraogo, I., Pierre G., (2010). Propriétés Nutritionnelles et Thérapeutiques du Fruit de Detarium microcarpum Guill. et Perr Nutritional and Therapeutic properties of the Fruit of Detarium microcarpum Guill. \& Perr. Fruit. Vegetable and Cereal Science and Biotechnology, 4(1), 26-30.

12. Ghedadba, N., Bousselsela, H., Hambaba, L., Benbia, S., Mouloud, Y. (2014). Évaluation de l'e activité antioxydante et antimicrobienne des feuilles et des sommités fleuries de Marrubium vulgare L. Phytothérapie, (12), 15-24.

13. Habibou H.H., Moutari S.K., Lawaly M.M., Idrissa M., Rabani A., Khalid I. (2018). Criblage phytochimique et dosage des polyphénols du Detarium microcarpum Guill. \& Perr. utilisé dans le traitement des maladies parasitaires au Niger. Afrique science, 14(5), 390-399.

14. Ikhiri, K. \& Ilagouma, A.T. (1995). fitoterapia, 66, 274.

15. Khoudali, S., Benmessaoud D., Essaqui1, A., Zertoubi M., Azzi M., Benaissa M. (2014). Étude de l'activité antioxydante et de l'action anti corrosion de l'extrait méthanolique des feuilles du palmier nain (Chamaerops humilis L.) du Maroc. Journal of Materials and Environmental Science, 5 (3), 887-898.

16. Kouyaté, A.M and Van Damme, P. (2002). Caractères morphologiques de Detarium microcarpum Guill. et Perr. au sud du Mali, Fruits, 57 (4), 231-238.

17. Kouyaté, A.M. (2005). Aspects ethnobotaniques et étude de la variabilité morphologique, biochimique et phénologique de Detarium microcarpum Guill. \& Perr. au Mali. Thèse de Doctorat, Université de Gand, Belgique. 207 p.

18. Ladoh Yemeda, C.F., Dibong, S.D., Nyegue, M.A., Djembissi, R.P., Lenta N.B., Mpondo M.E., Yinyang, J., Wansi, J.D. (2014). Activité antioxydante des extraits méthanoliques de Phragmanthera capitata (Loranthaceae) récoltée sur Citrus sinensis. Journal of Applied Biosciences, 84, 7636-7643.

19. Lee, K.W., Kim, Y.J., Lee, H.J., Lee, C.Y. (2003). Cocoa has more phenolic phytochimicals and a aigher Antioxydant capacity than theas and red wine. Journal of Agriculture and Food Chemistry. (3), 72927295.

20. Loubaki B.C., Ouattara, A. S., Ouattara C. A., Ouedraogo, R., Traore, A. S. (1991). Activités antimicrobiennes des extraits aqueux totaux de Detarium microcarpum [Cesalpinaceae (Gull et Perr)] sur huit 
espèces bactériennes impliquées dans certaines maladies infectieuses au Burkina Faso. Science et médecine, 66-73.

21. Mamta, S., Jyoti, S., Rajeev, N., Dharmendra, S. \& Abhishek, G. (2013). Phytochemistry of Medicinal Plants. Journal of Pharmacognosy and Phytochemistry, 1(6) 168-182.

22. Mounira, A. \& Mahdia, H. (2015). Contribution à l'étude phytochimique, les activités biologiques (Antioxydante et Antibactérienne) d'une plante médecinale Cleome arabica L (Région d'Oued Souf). Mémoire de Master. Universite Echahid Hamma Lakhdar D’el-Oued, (Algerie), 106 p.

23. Okolo, C.E, Akah, P.A and Uzodinma, S.U. (2012). Antidiabetic activity of root extract of Detarium microcorpum. (Fabiacase) Guill \& Perr. Phytopharmacology, 3(1), 12-18.

24. OMS (2002). Perspectives politiques de l'OMS sur les médicaments. Médecine Traditionnelle : Besoins croissants et potentiel. Genève.

25. Rakiatou T. (2014). Etude mécanique de la relation groupe chimiqueactivité antioxydante des extraits d'écorces de tronc de Khaya senegalensis A. Juss (Meliaceae). Thèse de doctorat, Université de Ouagadougou, (Burkina Fasso), 136 p.

26. Rouamba, A., Ouedraogo, M. \& Kiendrebeogo, M. (2017). Antioxidant capacity and genoprotective effect of ethanol fruit extract from Detarium microcarpum Guill. \& Perr.(Caesalpiniaceae). Asian Pacific Journal of Tropical Biomedicine, 7(1), 32-36.

27. Siddiqui, N., Abdur Rauf, M.D., Abdul Latif, M.D. \& Zeenat Mahmood, M.D. (2017). Spectrophotometric determination of the total phenolic content, spectral and fluorescence study of the herbal Unani drug Gul-e-Zoofa (Nepeta bracteata Benth). Journal of Taibah University Medical Sciences, 12(4), 360-363.

28. Tirichine H.S. (2010). Etude ethnobotanique, activité antioxydante et analyse phytochimique de quelques cultivars de palmier dattier (Phoenix dactylifera L.) du Sud-Est algérien. Mémoire de Magister en Biologie. Université d'Oranes-Es Senia, Oran, (Algérie), 88 p.

29. Valko, M., Rhodes, C., Moncol, J., Izakovic, M., Mazur, M. (2006). Free radicals, metals and antioxidants in oxidative stress-induced cancer. Chemico-Biological Interactions, 160, 1-40. 